\title{
Pleural Effusion Secondary to Obstructive Uropathy: A Case of Urinothorax
}

\author{
Andreia Freitas, Telmo Coelho, Sara Beça, Tiago Gregório \\ Internal Medicine Department, Centro Hospitalar Vila Nova de Gaia/Espinho, Vila Nova de Gaia, Portugal
}

Received: 07/03/2020

Accepted: $19 / 07 / 2020$

Published: 16/09/2020

How to cite this article: Freitas A, Coelho T, Beça S, Gregório T. Pleural effusion secondary to obstructive uropathy: a case of urinothorax. EJCRIM 2020;7: doi:10.12890/2020_001865.

Conflicts of Interests: The Authors declare that there are no competing interests.

Acknowledgements: We are grateful to Joana Mascarenhas for her assistance.

This article is licensed under a Commons Attribution Non-Commercial 4.0 License

\section{ABSTRACT}

Urinothorax is a rare type of pleural effusion and usually the result of genitourinary tract disease. An accurate and early diagnosis is crucial as resolution of the underlying pathology is the mainstay of treatment. We report the case of a 69-year-old man who was admitted to the Internal Medicine ward due to obstructive acute kidney injury of unknown origin. The patient was submitted to urinary catheterization and to right percutaneous nephrostomy. Two weeks after admission he developed a large left pleural effusion; a left urinoma was also visible on computed tomography. After thoracentesis, pleural fluid analysis demonstrated a paucicellular transudate with $\mathrm{pH}<7.40$ and pleural fluid/serum creatinine ratio $>1$.0. The diagnosis of urinothorax was made and further study allowed the diagnosis of prostate cancer as the aetiology of the obstruction. When bilateral percutaneous nephrostomy was performed, resolution of the urinothorax and normalization of renal function occurred.

\section{LEARNING POINTS}

- Urinothorax is a rare and possibly under-recognized cause of transudative pleural effusion; over the last five decades, fewer than 60 cases have been reported in the English literature worldwide.

- Diagnosis of urinothorax depends upon a high index of clinical suspicion and on pleural fluid analysis, but imaging can also support the diagnosis, for example by showing the presence of a urinoma.

- Clinicians should recognize urinothorax early in its clinical course as favourable outcomes are often achieved after correction of the underlying genitourinary tract pathology.

\section{KEYWORDS}

Pleural effusion, thoracentesis, hydronephrosis, urinoma, acute kidney injury

\section{INTRODUCTION}

Urinothorax is a rare and underdiagnosed cause of pleural effusion, typically a transudate. It can occur at any age and it is more frequent in males ${ }^{[1]}$. The accumulation of urine in the pleural space is usually the result of: (a) bilateral urinary obstruction; (b) genitourinary tract trauma; or (c) iatrogeny (for instance after nephrostomy tube placement, renal biopsy or lithotripsy) ${ }^{[2]}$. Diagnosis of urinothorax relies on a high index of clinical suspicion and on analysis of pleural fluid ${ }^{[3]}$. The repair of the underlying genitourinary tract disease is the mainstay of treatment ${ }^{[2]}$. Evacuating thoracentesis is recommended in patients with mild symptoms and placement of a chest tube is recommended for extremely large pleural effusions and very symptomatic patients ${ }^{[4]}$. 


\section{CASE DESCRIPTION}

We report the case of a 69-year-old man with a history of epilepsy, arterial hypertension and dyslipidaemia who presented to the emergency department after a seizure. The patient had been treated at home with ciprofloxacin for a urinary tract infection. The patient denied any complaints and vital signs were normal. Laboratory tests were notable for a creatinine of $2.44 \mathrm{mg} / \mathrm{dl}$ and urea $134 \mathrm{mg} / \mathrm{dl}$; urinalysis was normal. A renal ultrasound showed bilateral hydronephrosis of unknown aetiology. The patient was admitted to the Internal Medicine ward due to obstructive acute kidney injury. During hospitalization, the patient had progressive worsening of renal function (worst creatinine score: $5.66 \mathrm{mg} / \mathrm{dl}$ ) that only partially improved after urinary catheterization. Therefore, 2 weeks after admission a right percutaneous nephrostomy was performed. The procedure was uneventful; however, shortly afterwards, the patient developed shortness of breath without associated cough or chest pain. On clinical examination, the patient was apyretic and haemodynamically stable but tachypnoeic (28 breaths/minute) and with hypoxaemia $\left(\mathrm{PaO}_{2} 70.7 \mathrm{mmHg}\right)$. Chest examination showed dullness to percussion, absence of breath sounds and decreased transmission of vocal sounds in the left hemithorax. Chest radiography showed opacification of the entire left hemithorax with contralateral deviation of the cardiac silhouette, suggestive of large volume pleural effusion (Fig. 1).

Computed tomography of the chest confirmed the presence of a large left pleural effusion with complete atelectasis of the lung; additionally, a collection was observed in the region of the posterior perirenal space in contact with the posterior aspect of the renal pelvis, suggestive of a urinoma (Fig. 2).
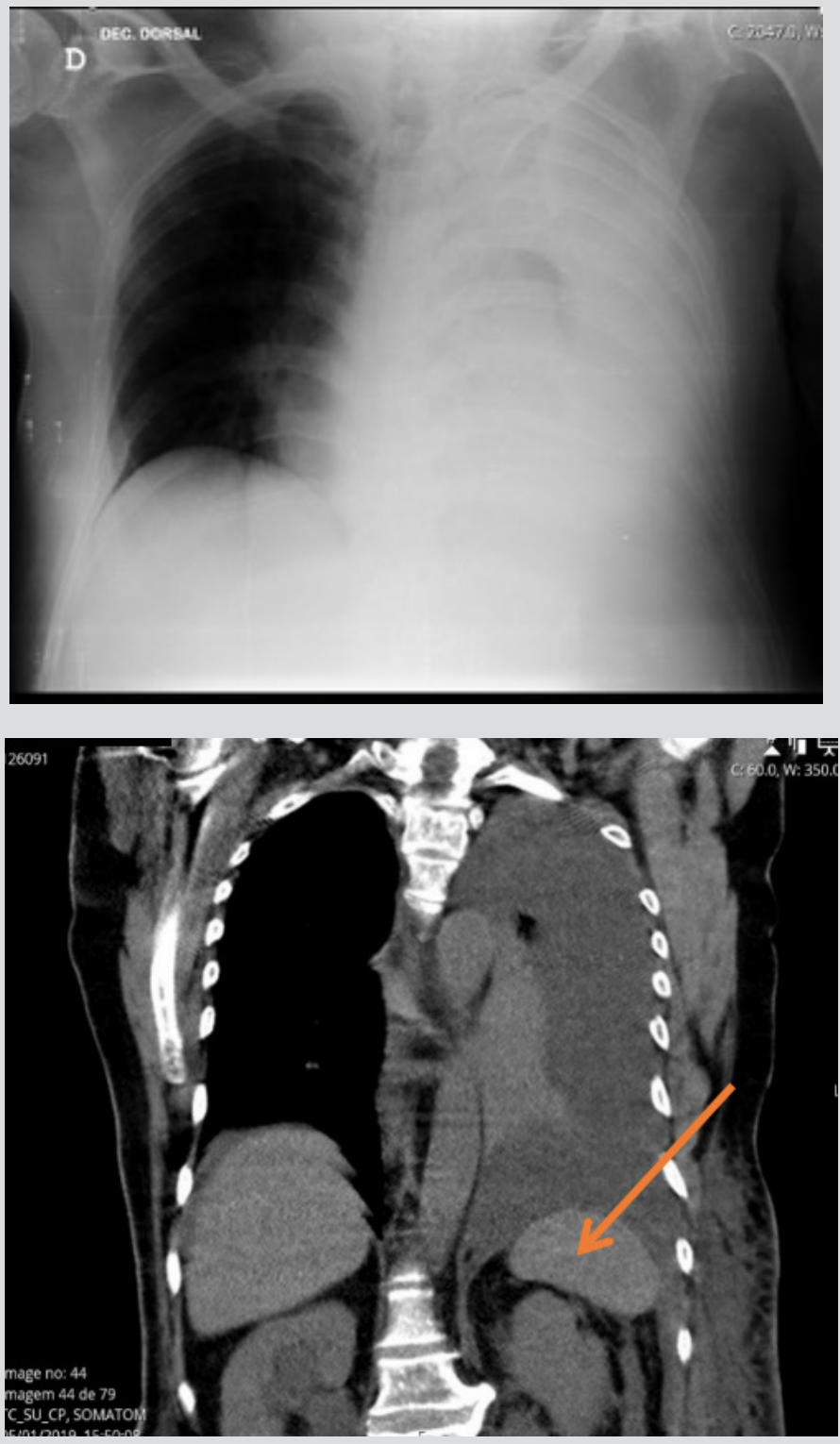

Figure 1. White lung. Chest radiography showed left pleural effusion

Figure 2. Urinoma. Computed tomography of the chest showed left pleural effusion with complete atelectasis of the lung; urinoma is present in the posterior perirenal space (orange arrow) 
A diagnostic and evacuating thoracentesis was performed with collection of 1,100 $\mathrm{ml}$ of clear yellow-citrus liquid. Pleural fluid analysis demonstrated $\mathrm{pH} 7.0$, glucose $139 \mathrm{mg} / \mathrm{dl}$, creatinine $6.12 \mathrm{mg} / \mathrm{dl}$ (pleural fluid/serum ratio: 1.18), lactate dehydrogenase $39 \mathrm{U} / \mathrm{l}$ (pleural fluid/serum ratio: 0.17), proteins $0.6 \mathrm{~g} / \mathrm{dl}$ (pleural fluid/serum ratio: 0.08 ) and albumin $0.3 \mathrm{~g} / \mathrm{dl}$ (pleural fluid-serum gradient: 0.075 ); pleural

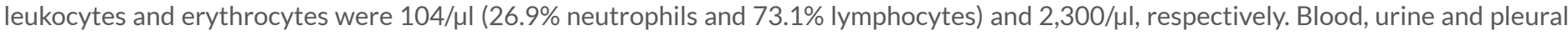
fluid cultures were sterile; cytology was negative for malignant cells. The diagnosis of urinothorax in the context of obstructive nephropathy was made and an additional left percutaneous nephrostomy was performed. Dyspnoea and hypoxaemia improved; progressive resolution of the pleural effusion and normalization of renal function were obtained. Further study done during hospitalization revealed elevated prostate specific antigen and a very hard prostate; a biopsy of the prostate was performed and the result showed a prostatic adenocarcinoma (Gleason 7). The patient was discharged from the hospital with bilateral nephrostomy 4 weeks after admission; follow-up was provided on an outpatient basis. To date, no recurrence of pleural effusion has been observed.

\section{DISCUSSION}

Our patient developed urinothorax due to bilateral obstruction of the genitourinary tract caused by prostate cancer. In a systematic review of 78 studies of urinothorax ${ }^{[5]}, 24 \%$ of the cases were due to obstructive uropathy. Two mechanisms are proposed for the pathophysiology of urinothorax: (a) translocation of urine directly through diaphragmatic pores due to increased retroperitoneal or intraperitoneal pressure; and (b) when a urinoma, resulting from vesicoureteral reflux secondary to high-pressure retention of urine, drains via communication between retroperitoneal and pleural lymphatics ${ }^{[2]}$, as in the present clinical case.

In our case, the first percutaneous nephrostomy was placed on the contralateral side of the pleural effusion and no traumatic placement of the inner end of the catheter was recognized.

Diagnostic criteria are not fully established in the literature, but urinothorax is most often a clear yellow paucicellular transudate, predominately mononuclear, with a pleural fluid/serum creatinine ratio $>1.0$ - the most specific biochemical feature ${ }^{[2]}$. It is the only transudate associated with $\mathrm{pH}<7.40^{[2]}$. As the basic characteristics of pleural effusion are inconsistent, one should have a high index of suspicion in the appropriate context. In this clinical case, bilateral hydronephrosis with worsening renal function and further evidence of rapid accumulation of pleural effusion led to clinical suspicion of urinothorax. This was confirmed by pleural fluid analysis, which demonstrated a transudate with $\mathrm{pH}<7.40$ and pleural fluid creatinine 1.18 times higher than serum creatinine. An accurate and early diagnosis is crucial as relief of genitourinary obstruction is the mainstay of treatment ${ }^{[2]}$. When appropriate urological diversion (bilateral percutaneous nephrostomy) was performed, resolution of the urinothorax occurred.

\section{REFERENCES}

1. Kelley M, Spieler B, Rouse C, Karl B, Marshall R, Carbonella G. Urinothorax: a rare complication of percutaneous nephrostomy. Radiol Case Rep 2019;14(6):729-733.

2. Austin A, Jogani AN, Brasher PB, Argula RG, Huggins JT, Chopra A. The urinothorax: a comprehensive review with case series. Am J Med Sci $2017 ; 354(1): 51-59$.

3. Batura D, Haylock-Vize P, Naji Y, Tennant R, Fawcett K. Management of iatrogenic urinothorax following ultrasound guided percutaneous nephrostomy. J Radiol Case Rep 2014;8(1):34-40.

4. Ferreira PG, Furriel F, Ferreira AJ. Urinotórax como causa rara de derrame pleural - revisitação a propósito de caso clínico. Rev Port Pneumol 2013;19(2):80-83.

5. Toubes ME, Lama A, Ferreiro L, Golpe A, Álvarez-Dobaño JM, González-Barcala FJ, et al. Urinothorax: a systematic review. J Thorac Dis 2017;9(5):1209-1218. 